\title{
Lidil
}

Revue de linguistique et de didactique des langues

$57 \mid 2018$

Démarches créatives, détours artistiques et appropriation des langues

\section{Mother Tongue Other Tongue : approche créative des langues pour une (ré)appropriation linguistique}

Mother Tongue Other Tongue: A Creative Approach to Languages for Linguistic (Re)Appropriation

\section{Malika Pedley}

\section{OpenEdition Journals}

Édition électronique

URL : http://journals.openedition.org/lidil/4820

DOI : 10.4000/lidil.4820

ISSN : $1960-6052$

Éditeur

UGA Éditions/Université Grenoble Alpes

\section{Édition imprimée}

ISBN : 978-2-37747-048-8

ISSN : $1146-6480$

\section{Référence électronique}

Malika Pedley, "Mother Tongue Other Tongue : approche créative des langues pour une

(ré)appropriation linguistique », Lidil [En ligne], 57 | 2018, mis en ligne le 01 mai 2018, consulté le 01 mai 2019. URL : http://journals.openedition.org/lidil/4820; DOI : 10.4000/lidil.4820

Ce document a été généré automatiquement le 1 mai 2019.

(C) Lidil 


\section{Mother Tongue Other Tongue: approche créative des langues pour une (ré)appropriation linguistique}

Mother Tongue Other Tongue: A Creative Approach to Languages for Linguistic (Re)Appropriation

\section{Malika Pedley}

\section{Introduction}

1 Mother Tongue Other Tongue est un concours de poésie ouvert à toutes les langues, proposé dans les écoles primaires et secondaires en Écosse. Enquêtes à l'appui, nous montrerons dans cet article en quoi ce projet se présente comme une opportunité d'appropriation ou de réappropriation linguistique pour l'enfant. Après avoir présenté les enjeux et principes de ce concours, nous apporterons quelques éléments de définition à la notion d' appropriation. Une fois notre corpus d'analyse défini en deuxième partie, nous expliquerons en quoi ce projet induit un processus d'autonomisation de la part de l'enfant. Enfin, nous nous intéresserons à ce projet en tant qu'exercice de médiation en montrant comment il participe à l'appropriation linguistique.

\section{Contexte et notions}

\subsection{Un concours de poésie multilingue en Écosse}

2 Mother Tongue Other Tongue (désormais MTOT) est un concours annuel de poésie multilingue proposé aux enfants d'école primaire et secondaire dans différentes parties du Royaume-Uni. L'enquête dont cet article fait état s'est consacrée à l'édition 2015-2016 de MTOT en Écosse ${ }^{1}$. Pour participer au concours, l'élève propose une production bilingue composée d'un poème ${ }^{2}$ en langue autre que l'anglais accompagné d'un texte explicatif en 
anglais. Il y a deux catégories d'entrée : Mother Tongue accueille des poèmes écrits dans une langue connue par un biais autre que l'école; Other Tongue, des poèmes écrits dans une langue que l'enfant pratique ou apprend à l'école. En valorisant les identités plurilingues locales, l'objectif est de promouvoir l'apprentissage des langues auprès de l'ensemble des écoliers. Ce concours œuvre pour une vision plus positive du plurilinguisme au niveau individuel et collectif.

MTOT s'inscrit dans une démarche originale à l'école, de par la place accordée aux langues autres que l'anglais et par un rapport non normatif à l'écriture. Toute langue peut entrer dans ce concours, sans distinction aucune. Certaines ont une place relative à l'école, comme les langues vivantes étrangères (désormais l'équivalent britannique, MFL, Modern Foreign Languages, c'est-à-dire le français, l'espagnol, et plus rarement l'italien ou l'allemand) ou ponctuellement le gaélique et le scots. D'autres langues n'ont aucune place dans le curriculum de l'élève, c'est le cas par exemple de l'arabe, du somali, du malayalam ou du tchèque. Les deux catégories du concours (Mother Tongue et Other Tongue) séparent les entrées selon un mode d'apprentissage informel ou formel de la langue, ne faisant donc pas de distinction entre des langues données ou entre différents degrés de maitrise de celles-ci. Ainsi, par exemple, on trouve des poèmes en français dans chacune des deux catégories. Le gaélique est aussi la langue de scolarisation d'élèves ayant participé au concours et inscrits dans une école d'immersion. Ces élèves parlent couramment le gaélique mais leur contribution figure parmi la catégorie Other Tongue, car il s'agit d'une langue apprise en contexte scolaire.

L'élève est libre de composer son propre texte ou de reprendre celui d'un auteur. Le concours accepte tout type de textes pouvant s'apparenter à de la poésie, des comptines, des chansons, voire même du rap. Afin de bien différencier les deux parties de la production bilingue, nous conservons cette terminologie : le poème en langue autre que l'anglais et le texte explicatif en anglais. Retenons tout de même qu'il s'agit d'une écriture de forme totalement libre dans une langue qui n'est pas forcément connue des membres du jury ou du public. Ce décalage entre l'école dans laquelle l'enfant crée son poème et cette liberté de création est une des spécificités de ce concours, laissant libre cours à l'imagination et à la créativité de l'enfant. La poésie est présentée par les organisateurs du concours comme une forme d'écriture plus libre favorisant l'expression de l'enfant qui peut s'affranchir des questions de maitrise de la langue. Celle-ci n'est d'ailleurs ni un prérequis ni un objectif pédagogique.

\section{2. (Ré)appropriation linguistique : éléments de définition}

En didactique des langues, la notion d'appropriation est utilisée pour désigner un processus permettant à l'apprenant d'accéder à un usage personnel de la langue (Cuq \& Gruca, 2008): l'appropriation se caractérise par un processus d'acquisition qui est «naturel, implicite, inconscient», ou par l'apprentissage, un processus "artificiel, explicite, conscient » (p. 113). Ces deux types de processus, aussi distincts qu'ils puissent paraitre, exercent entre eux une influence mutuelle. Cette vision de l'appropriation s'inscrit dans une perspective didactique, où l'objectif est l'usage de la langue.

6 L'objectif de MTOT étant avant tout de libérer la parole de l'enfant à travers ses langues, nous nous rapprochons davantage de la façon dont Castellotti (2017) envisage la notion d'appropriation. Dans son récent ouvrage, elle pose d'emblée la nécessité de distinguer acquisition, apprentissage et appropriation des langues. L'appropriation d'une langue ne 
peut se résumer à l'idée de possession, car une langue est immatérielle. La notion dépasse aussi celle de maitrise, tant dans l'acception commune du terme (domination) que dans l'acception de compétence. La notion d'appropriation suppose plutôt l'idée d'une relation entre un individu, une langue et les autres. Appropriation et maitrise d'une langue ne vont pas de pair, bien qu'il paraisse évident que plus l'individu comprend la relation qui le lie à cette langue, plus il est conscient de ses objectifs en termes de niveau de compétences dans cette langue. De même, comme le montre Castellotti (2017), on peut tout à fait acquérir une certaine maitrise d'une langue sans forcément tisser de lien personnel avec celle-ci, sans se l'approprier.

7 Enfin, l'appropriation est à la fois le résultat et le processus d'une "expérience éminemment personnelle »:

Aucun [humain] ne s'approprie une nouvelle «langue » ni n'éprouve une nouvelle expérience linguistico-culturelle sur le même mode, car cette appropriation constitue une expérience à chaque fois différente, en fonction de son histoire et de ses projets. (Castellotti, 2017, p. 51)

8 L'expérience que propose MTOT à chaque élève est une opportunité de valoriser son répertoire linguistique et langagier. Elle constitue en soi une « expérience éminemment personnelle» (Castellotti, 2017, p. 48) des langues, avec en filigrane, un objectif d'exposition publique. Afin de transmettre à son public lecteur ou auditeur des éléments de son poème et de sa langue, l'enfant effectue un travail de médiation au travers principalement de son texte explicatif en anglais. Bien que d'autres domaines des sciences humaines comme la psychologie ou la sociologie peuvent l'éclairer, la notion de médiation est à entendre ici au sens didactique du Cadre européen commun de référence pour les langues (désormais, CECRL - Conseil de l'Europe, 2001), comme une des activités langagières que tout apprenant doit être amené à réaliser, à l'oral comme à l'écrit. Essentielle à la communication, elle a pour première fonction de donner à un locuteur l'accès à un texte qui lui est opaque, pour des raisons linguistiques ou langagières. Nous verrons que les stratégies de médiation utilisées par les enfants dans le concours MTOT dépassent les exemples proposés par le CECRL ${ }^{3}$.

9 Même si le projet aboutit à la constitution de productions majoritairement bilingues, le travail réalisé en amont en classe s'avère bien plus complexe, explorant l'ensemble du répertoire linguistique et langagier de l'enfant. Ce ne sont pas des compétences dans une langue précise qui sont sollicitées, mais plutôt la compétence plurilingue et pluriculturelle de l'enfant : l'élève prend conscience à travers cet exercice des ressources linguistiques et culturelles dont il dispose et de sa capacité à jouer de celles-ci (Coste, Moore \& Zarate, 1997).

Les parcours de vie des enfants participant au concours sont très divers, certains ayant connu des changements importants impactant leurs pratiques linguistiques. Lors de nos entretiens ${ }^{4}$, plusieurs enfants ont expliqué avoir eu le sentiment de délaisser leur langue première au profit de l'anglais, langue de scolarisation et langue commune de leur nouveau pays d'accueil. Pour ces enfants, le travail de MTOT implique alors de retrouver ou même de reconstruire un rapport à ces langues actuellement délaissées, invisibles à l'école. Pour être plus précis, dans certains cas où la biographie langagière de l'enfant a pu être mouvementée et certaines pratiques linguistiques occultées, on parlera de réappropriation linguistique. 


\section{Méthodes d'investigation}

11 L'enquête portant sur l'édition 2015-2016 de MTOT en Écosse s'est appuyée sur deux méthodes d'investigation. Neuf entretiens oraux semi-directifs ont été réalisés auprès de 49 élèves de Glasgow provenant d'une école primaire immersive en gaélique et de trois écoles primaires où le projet était encadré par une enseignante EAL (English as an Additional Language - anglais langue seconde) $)^{5}$. La transcription de ces entretiens constitue notre premier corpus. Dans un souci de représentativité, le deuxième corpus rassemble 97 productions bilingues : toutes celles de la catégorie Mother Tongue auxquelles ont été ajoutées les entrées en gaélique de la catégorie Other Tongue. Au total, le premier corpus fait état de 18 langues différentes utilisées par les 49 élèves enregistrés, le second recense 36 langues différentes.

12 Les langues les plus fréquemment recensées à travers nos deux corpus sont, dans l'ordre, le polonais (représentant un tiers de chacun des corpus), le gaélique, le mandarin, le français, l'ourdou, l'espagnol, le portugais, l'arabe, le swahili, le zoulou, l'allemand, le malayalam et le tagalog. Ces productions sont celles d'élèves d'école primaire et de quelques élèves du secondaire (11 productions).

\section{Processus d'autonomisation pour une réflexion sur les langues}

\subsection{Personnaliser son écriture}

Les témoignages recueillis lors des entretiens ont montré des réticences de nombreux élèves à l'égard du concours à ses prémisses. Ces élèves expliquent avoir ressenti une gêne à l'idée d'écrire un poème dans une langue qui n'est pas la langue commune en Écosse. L'analyse des séquences interactionnelles dans lesquelles les enfants racontent leur propre expérience d'écriture montre une évolution au fil des séances, cette gêne se dissipant.

SI. - I remember on that day after Miss $B^{*}$ told me I went home and I told my mum and she was like you do rea(lise?)/ $\mathrm{hm}$ this is the first time you've ever done this and I was like but I've never heard of the competition I had this ide/I had mixed feelings I was happy because I could share my language and I was upset because I couldn't actually find a poem and then it got to the weeks later it just popped into my head that poem and I just started writing it down.

\section{Ecole1 Dec2015 LU-SI ${ }^{6}$}

14 À travers ce témoignage, cette élève explique une gêne due à un manque de connaissances dans la langue et culture du poème. Une solution (demander de l'aide aux parents) ou bien un élément déclencheur provoqué par l'élève lui-même (une idée) permet de dépasser cette gêne, présentée comme un obstacle, et d'avancer dans le processus d'écriture. Dans l'extrait suivant, BL explicite cet élément déclencheur : peu à peu, l'élève prend conscience de la nécessité de s'investir pleinement dans le projet.

BL. - Well I was like ooooh what are we doing I was like what's the point of this like I don't get it and I was like I really don't want to do this but I don't know I like it because we got to draw and everything (laughter) but then I actually started liking it so it's good/ but first I didn't

M. - And then what do you think made you change your mind? 
BL. - Well I learned that we've just got to express ourselves and not like just do a

drawing do a poem like that actually meant something

M. $-\mathrm{Hmm}$

BL. - started enjoying the poems that's what changed

Ecole2 Dec2015 P6-P7 7

15

D'après ce témoignage, un changement d'attitude s'impose : s'exprimer de manière à faire sens (autrement dit, écrire quelque chose qui a du sens pour soi) et aimer son poème et l'écriture de son poème. Elle oppose cette nouvelle attitude à celle consistant à exécuter une consigne donnée (faire un dessin, faire un poème). De manière générale, on note une prise de responsabilité et une implication personnelle. L'exercice amène l'élève à se livrer publiquement sur un sujet très personnel, une langue ignorée à l'école ou, dans le cas du gaélique, peu présente dans des instances de socialisation hors terrain scolaire. En termes d'expression, l'enfant doit pouvoir personnaliser son écriture, indépendamment de la norme scolaire.

\subsection{Prendre position en tant qu'auteur}

Dans un article consacré à l'étude de dessins réflexifs d'enfants pour comprendre leur appréhension de la pluralité linguistique, Castellotti et Moore (2009) expliquent : «[Les enfants] sont, en effet, en position d'auteurs, qui se réapproprient leur dessin et vont proposer des clés pour en construire, en interaction, une interprétation. » (p. 45) L'étude des textes explicatifs montre une position affirmée d'auteur, qui contraste avec le malaise ressenti en début d'expérience et évoqué par les élèves lors des entretiens.

Dans les entretiens, cette posture est perceptible lorsque l'élève raconte son expérience d'écriture. Nombreux sont les élèves qui ne maitrisent pas la norme graphique de la langue du poème. Une des solutions est alors d'inventer son propre code :

SI. - I found it was great because it was actually my first time spelling in my own language and I remember I was at home and my mum was in the bedroom and I was just thinking of using syllables and spelling it and I was like mum is that right and she was like how did you know how to spell that I was like I just used syllables LU. - That's the same with my language because nobody knows how to write it in my house [...] so I just thought why don't just use English letters to spell the word Ecole1 Dec2015 LU-SI ${ }^{8}$

Enfin, même si le projet se pérennise en une production écrite plurilingue, les élèvesauteurs ont été amenés à en rendre compte à l'oral tout au long du concours : lecture et récitation des poèmes dans les groupes d'ateliers MTOT, lors d'assemblées générales au sein des écoles et lors de la cérémonie de remise des prix.

\subsection{Se situer par rapport aux autres}

Bien que MTOT implique un travail éminemment personnel, l'enfant puise ses forces dans les différentes relations qu'il entretient: soutien, apport de connaissances de certains, regard approbateur d'autres et constitution d'un groupe social fédéré par le projet.

Dans chaque école participant au concours, un(e) enseignant(e) est en charge du projet. Nos entretiens réalisés en présence de l'enseignante ont montré une implication importante de celle-ci dans le projet, sa place dialogique alternant entre celle d'intervieweuse à mes côtés et celle de participante du côté des élèves (Audras, Bigot \& Vasseur, 2013, p. 249). Les élèves, aussi, ont témoigné du soutien de l'enseignante dans la 
réalisation de leurs travaux. Cependant, ce soutien a pu être limité en ce qui concerne l'apport linguistique et culturel lorsqu'il s'agissait d'une langue de famille.

21 Pour que les enfants participent au concours, les enseignants en charge ont dû au préalable obtenir l'accord de leurs parents. Certains parents ont tenu à la participation de leur enfant au concours, comme l'explique ici cet élève de manière potentiellement exagérée : «my mum kept on shouting at me to do $i t^{9}$ » (KE1, Ecole3 Dec2015 P5-P6). Les parents se sont avérés de véritables référents culturels en proposant des poèmes de leur répertoire et des référents linguistiques en aidant l'enfant dans l'écriture et la traduction du poème. L'implication des parents a pu s'avérer essentielle pour la réalisation du projet personnel de l'enfant. En outre, d'un point de vue symbolique, ce soutien contribue à reconnaitre la légitimité du projet aux yeux de l'enfant.

Enfin, le regard des camarades ne prenant pas part au concours s'avère de toute importance pour les participants. Lors des entretiens, plusieurs élèves ont fait part de commentaires positifs de leurs pairs à l'égard du concours, ce qui a pu les encourager. Dans l'extrait suivant, l'élève fait part d'une discussion avec ses pairs dans laquelle elle les incite à s'interroger sur leur propre compétence plurilingue. Pour elle, en apprenant l'espagnol à l'école, tout enfant peut participer à ce projet plurilingue :

SI. - [people in class] were like where are you going I told them it's this project about people who speak more than one language and then they were like oh that's cool and I was like why don't you guys join in and they were like oh because I don't speak another language but I was like you do realise we learn Spanish in school you could find a Spanish poem but they're like no I don't wanna do it and they were like well that's great because we've never heard you speaking your language so to get a chance to listen to you saying a poem that's really great.

$$
\text { Ecole1 Dec2015 LU-SI }{ }^{10}
$$

L'enseignante que nous avons rencontrée a conscience de l'influence remarquable des pairs sur les élèves plurilingues. La tenue d'une assemblée générale dans l'école autour des poèmes produits est d'ailleurs un moyen explicite de confronter l'ensemble des élèves à cette réalité multilingue. Ainsi, MTOT, à travers son ambition globale, permet d'ancrer une réflexion locale.

$24 \mathrm{Au}$ sein des ateliers, les enfants ont pu se livrer, dire leur poème dans leur langue et échanger autour de leur point commun, l'existence d'une langue autre que l'anglais dans leur répertoire. Dans une dynamique d'expressivité et de créativité, ont pu naitre une confiance et un sentiment d'appartenance à une communauté nouvelle. Comme le montre l'extrait suivant, cette situation nouvelle contraste avec le reste du temps scolaire :

KE1. - always people ask me to speak in my language but I don't want to because

I'm scared and afraid

K. - What are you scared and afraid of?

KE1. - Because people think what is it/ just/ you know/ like [...] you just ask in

English instead of your language it's just/ you just feel scared [...]

K. - What about with us here?

KE1. - I'm confident here because it's people but in class I'm not Ecole3 Dec2015 P5-P6 ${ }^{11}$

Cette reconnaissance de soi dans l'autre dépasse le cadre du groupe de travail MTOT et s'étend aux situations plurilingues en général. Ainsi, dans les deux extraits suivants, deux élèves distinctes (l'une locutrice d'edo, l'autre de gaélique) font preuve d'empathie, en comparant chacune sa situation à celle d'un locuteur d'une autre langue (respectivement le polonais et le français) :

M. - How did it feel to talk about and to speak in Edo at school? 
LU. - It feels ... just normal [...] like speaking Polish [...] I don't know speaking

Polish in school like if you are like Polish is ... it just feels normal to me [...]

Ecole1 Dec2015 LU-SI ${ }^{12}$

So. - it's not different at all/ if you're like French for example and you haven't spoken any English and then you come and move here and you speak English like/

in France they'd be like oh is it any different but it's not [...]

Ecole4 Avr2016 P7- $1^{13}$

2015, p. 22-23), à laquelle adhèrent les élèves participant au projet. Les membres de cette communauté se reconnaissent en elle du fait que plusieurs langues sont présentes dans leur répertoire linguistique et que lorsqu'ils se retrouvent, ils peuvent s'exprimer au sujet de celles-ci et les exposer en les pratiquant dans la confiance d'être respectés et écoutés. MTOT permet ainsi l'émergence d'une communauté plurilingue. Nous préférons cette appellation à celle de communauté multilingue qui, bien qu'elle indique la présence de plusieurs langues dans un milieu, ne spécifie pas la nécessité pour chaque membre d'être plurilingue et de pratiquer l'ensemble de ses langues. Cette communauté se définit avant tout en termes de communauté de pratique au sens linguistique, puisque ce sont les pratiques plurilingues effectives en son sein qui la régissent. Il s'agit d'une communauté imaginée (Anderson, 1991, p. 6) puisqu'elle se crée avec et par les participants, d'abord de manière très concrète à l'échelle des ateliers puis par extrapolation à l'échelle de l'ensemble des participants au concours, par imagination, puisque tous ne se rencontreront pas mais sont en «communion » les uns avec les autres par ce qu'ils ont construit.

\section{Médiation : transmission et appropriation}

En faisant référence au CECRL, Cavalli et Coste (2015) définissent la médiation comme une action qui tend à "réduire l'écart entre deux pôles distants ou en tension» (p.13). Toujours d'après le CECRL, une telle opération se concrétise notamment par une « reformulation écrite ou orale, à destination d'un ou de plusieurs tiers, d'un texte, oral ou écrit, auquel ces tiers n'ont pas accès direct » et cette reformulation est réalisée «à des fins de transmission » (Cavalli \& Coste, 2015, p. 28).

Dans le cas de MTOT, l'action de médiation est rendue évidente à travers le texte explicatif en anglais. Les " pôles distants ou en tension » dont il s'agit de réduire l'écart se présentent sur des plans différents. D'un point de vue formel, la médiation s'exerce entre deux langues, mais aussi deux genres textuels. D'un point de vue interactionnel, elle s'exerce entre l'élève et son public et à une échelle plus large, entre deux communautés, la communauté plurilingue et une communauté englobante majoritairement anglophone.

Nos analyses du corpus de productions bilingues ont permis de mettre en évidence l'utilisation de six stratégies de médiation: l'illustration, la traduction et la translittération se situent en marge du texte explicatif; la reformulation, l'explicitation et la narration constituent le corps de celui-ci ${ }^{14}$.

Le corpus de productions bilingues donne à voir un usage généreux des fonctions informatiques à disposition des élèves pour personnaliser en couleurs et en images leurs travaux. Cependant, très peu ont une fonction de médiation et la majorité reste somme toute décorative (frises de cœurs, smileys et animaux variés). Quelques exemples se 
démarquent, comme la photographie de la figure 1 qui permet un accès visuel à l'anone, fruit exotique peu connu en Écosse.

Figure 1. - Production bilingue anglais/bengali ${ }^{15}$.

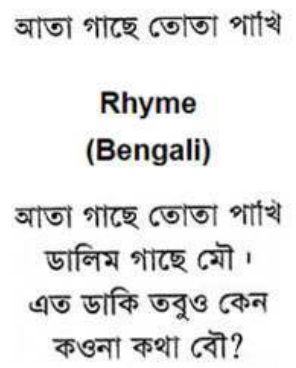

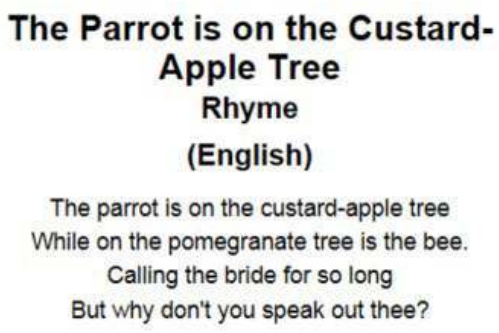

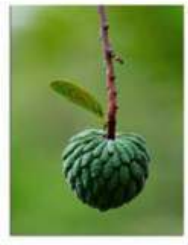

My poem is a rhyme about a parrot sitting in a custard apple tree. This rhyme is very popular among the children in Bangladesh. Custard apple is a fruit from India and is one of my Mum's favourite fruits. My Mum use to tell me this rhyme when I was younger at bedtime and when we visit my Grandma in Bangladesh, she tells me it then too.

My favourite part of the poem is when the parrot is shouting out to the bride. When I heard the poem it makes me think about the parrot sitting in the tree. I wonder if the parrot is singing something to the bride.

Courtesy SCILT

31 La traduction fait partie des exemples de stratégies de médiation citées par le CECRL (Cavalli \& Coste, 2015, p. 30). Alors qu'elle ne faisait pas partie des exigences du concours, environ $30 \%$ des poèmes présents dans notre corpus sont accompagnés d'une traduction. Cet exercice permet une médiation de texte à texte la plus fidèle possible. Si elle est autorisée, elle ne semble pas exclusive comme outil de médiation, car seules huit productions de notre corpus n'utilisent que la traduction comme texte en anglais. Les autres l'utilisent en supplément du texte explicatif en anglais, comme en figure 1.

Comme nous l'avons déjà mentionné, certains enfants n'ayant pas accès à la graphie de la langue du poème inventent leur propre code graphique, une translittération ${ }^{16}$ du poème. Ils font ainsi preuve d'inventivité et de créativité tout en mettant à profit leurs compétences métaphonétiques grâce à leur capacité en anglais. Cette stratégie ne doit en revanche pas être considérée comme une voie de recours pour pallier un manque de compétences à l'écrit : en effet, certaines productions bilingues donnent à voir le poème écrit dans sa graphie d'origine accompagnée de sa translittération. La figure 2 montre un extrait de production en trois versions : le poème écrit selon la norme graphique de la langue source (malayalam), sa traduction en anglais, et entre les deux, une version intermédiaire du poème en malayalam transcrite par l'élève selon son appréhension phonétique de l'anglais. En tant que stratégie de médiation, la translittération s'avère un outil précieux à l'écrit. En effet, dans le cas d'une diffusion écrite du poème, elle est la seule action permettant au lecteur d'en imaginer les sonorités. En outre, elle constitue une aide importante pour certains élèves-auteurs qui ne maitrisent pas (encore) suffisamment la norme graphique de la langue du poème pour pouvoir la lire avec aisance. 
Figure 2. - Production bilingue malayalam/anglais.

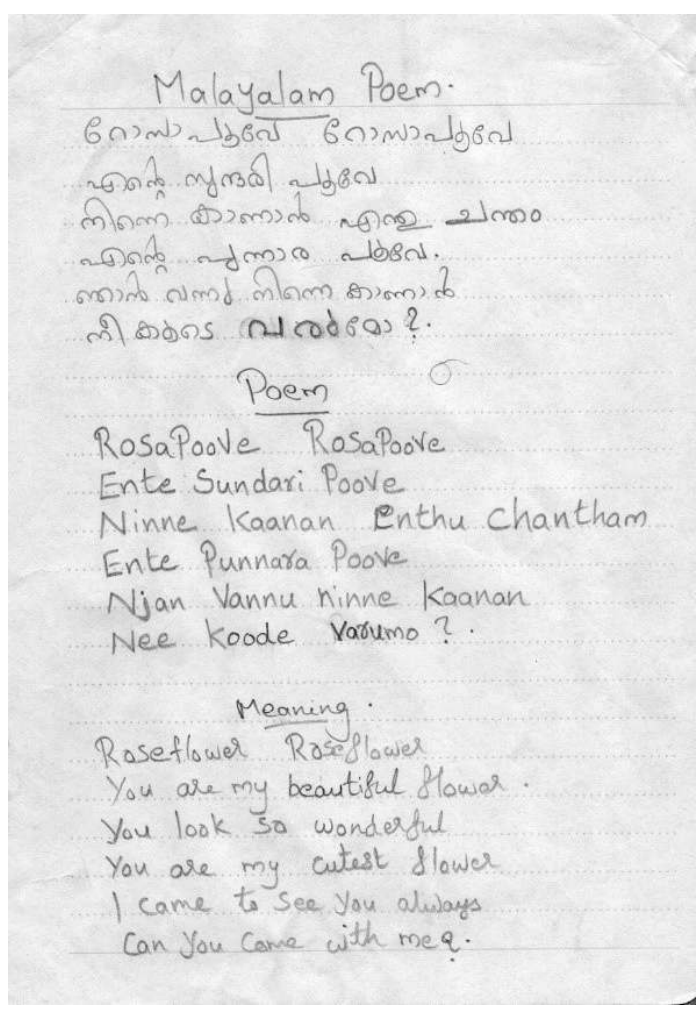

Courtesy SCILT

Environ $90 \%$ des textes explicatifs de notre corpus commencent par une brève reformulation du thème du poème ou de ce qui semble à l'élève être le plus probant dans son poème. En utilisant ses propres mots, l'élève-auteur s'approprie le texte. En sélectionnant certains éléments du poème, l'élève choisit ce qu'il transmet. La première phrase du texte explicatif en figure 1 reformule la thématique globale du poème.

Au cours du texte explicatif, il est fréquent que l'enfant choisisse d'expliciter un passage du poème. L'enfant cite alors le passage directement dans la langue d'origine, le traduit ou le reformule, puis l'explicite. Le deuxième paragraphe du texte explicatif de la figure 1 fait l'objet d'une explicitation d'un passage ici reformulé.

Enfin, la narration constitue un élément complexe du texte explicatif. À travers leur texte en anglais, les enfants racontent des faits, des anecdotes qui sont autant d'éléments contextuels permettant de mieux appréhender à la fois le poème et la relation qu'entretient l'élève avec celui-ci. En position d'expert, l'enfant apporte des éléments culturels ou linguistiques qui permettent de mieux comprendre le contexte du poème. À cela s'ajoutent des éléments beaucoup plus personnels : le poème renvoie de nombreux élèves à des expériences de vie précises qu'ils racontent. Mettant souvent en scène des membres de la famille et des territoires d'origine ou d'adoption, l'enfant ancre son poème dans sa propre histoire de vie. Le texte explicatif de la figure 1 illustre tout à fait ces vaet-vient entre différents territoires et entre récit du poème et de son auteur : l'enfant nous donne d'abord des éléments contextuels propres au poème (sa popularité et des renseignements concernant le fruit dont il s'agit); puis, il donne des éléments plus personnels (le fruit préféré de sa mère et le lien familial avec ce poème). Dans cette dernière partie, l'enfant évoque un temps révolu (" when I was younger at bedtime ») et un 
univers familial visité régulièrement, celui des vacances auprès de sa grand-mère au Bengladesh.

Le travail de médiation que suscite l'élaboration du texte explicatif implique de la part de l'élève la mise en mots d'une réflexion métalinguistique et métaexpérientielle (Dompmartin-Normand, 2016, p. 237). L'enfant construit alors un récit autour de son poème et se l'approprie.

37 La pratique de l'écrit peut s'avérer ludique, inventive et libératrice. Dans son article consacré aux pratiques d'écriture " clandestines ${ }^{17}$ » d'une enfant dans une langue bricolée (supposée être le « chinois »), Moore (2005) démontre un impact considérable de celles-ci lorsqu'elles sont régulières sur la construction de l'identité linguistique. La translittération, intermédiaire graphique également "bricolé », est un moyen pour l'élève de renouer avec sa langue en la pratiquant et en créant par son biais. À travers la narration, l'élève écrit son lien avec son poème, sa langue et sa culture. Par le biais d'un exercice d'écriture créative ${ }^{18}$ en langues, l'enfant est amené à réfléchir aux relations qu'il entretient avec les langues de son répertoire. L'appropriation ou la réappropriation linguistique se manifeste alors à travers le discours de l'enfant et les éventuels projets qu'il construit avec cette langue. Ces derniers sont discutés en conclusion.

\section{Conclusions}

Si l'on reprend l'objectif premier du concours, promouvoir l'apprentissage des langues au Royaume-Uni, cette (ré)appropriation nous semble une étape tout à fait nécessaire. En effet, avant d'élaborer un plan de stratégie pour l'enseignement et l'apprentissage des langues à l'école, il importe de faire changer les mentalités générales vis-à-vis du plurilinguisme, c'est-à-dire, surtout, montrer l'intérêt, dans un pays anglophone, de parler et d'apprendre d'autres langues.

En assumant sa position d'auteur et en adhérant à la communauté plurilingue émergente, l'enfant assume ses pratiques plurilingues et ses compétences partielles. Il a conscience que ses ressources linguistiques ne se limitent pas à une dichotomie schématisée entre langue(s) de l'école et langue(s) de la maison, et il intègre dans son répertoire des langues apprises en tant que MFL ou rencontrées lors d'ateliers d'éveil aux langues. Il prend alors conscience de la richesse de son répertoire et du potentiel que représente sa compétence plurilingue et pluriculturelle. À l'issue du concours, l'appropriation ou la réappropriation de la langue du poème se manifeste notamment à travers une motivation accrue pour l'apprentissage de cette langue, mais aussi de création avec cette langue, de manière plus ou moins « bricolée », voire en mêlant les langues.

L'expérience MTOT a permis à ses participants d'ouvrir les yeux sur la diversité linguistique environnante tout en s'inscrivant au sein de celle-ci. Si le contexte de MTOT est propice à un travail d'appropriation de la langue, celui-ci se trouve intimement lié au sentiment d'appartenance à la communauté plurilingue émergeante. Ainsi, le parcours commun des participants à MTOT mène à un désir convergeant de projet collectif autour des langues: les élèves de plusieurs groupes ont manifesté l'idée de faire connaitre les langues du groupe au reste de l'école afin d'informer, sensibiliser et former leurs pairs aux enjeux du plurilinguisme. En ce sens, les participants eux-mêmes deviennent acteurs de la promotion du plurilinguisme et de l'apprentissage des langues. 
41 Les politiques éducatives écossaises envisagent qu'à partir de 2021, chaque élève apprenne deux langues en plus de sa langue maternelle au cours de son cursus primaire et secondaire (Scottish Government, 2012). Malgré la dynamique actuelle autour du multilinguisme latent, on ne peut prédire si les langues valorisées dans le cadre de MTOT pourront toutes l'être dans le cadre d'un enseignement. Il est probable que, devant le grand nombre de langues représentées, certaines ne bénéficient pas d'un enseignement. Aussi, il n'est pas impossible que le capital symbolique (Bourdieu, 1982) attribué aux langues reste un critère privilégié de choix, tant pour les écoles que pour les familles.

\section{BIBLIOGRAPHIE}

ANDERSON, Benedict. (1991). Imagined Communities: Reflections on the Origin and Spread of Nationalism (Revised edition). New York: Verso.

AUDRAS, Isabelle, BIGOT, Violaine \& VASSEUR Marie-Thérèse. (2013). Quand des élèves enquêtent sur les langues de leur école : retour sur un projet pédagogique. Dans V. Bigot et coll. (dir.), Vers le plurilinguisme ? 20 ans après (p. 339-353). Paris : Éditions des archives contemporaines.

BouRdieu, Pierre. (1982). Ce que parler veut dire : l'économie des échanges linguistiques. Paris : Fayard.

CASTEllotTI, Véronique. (2017). Pour une didactique de l'appropriation. Diversité, compréhension, relation. Paris : Didier.

CAstellotti, Véronique \& Moore, Danièle. (2009). Dessins d'enfants et constructions plurilingues. Territoires imagés et parcours imaginés. Dans M. Molinié (dir.), Le dessin réflexif. Éléments d'une herméneutique du sujet plurilingue (p. 45-85). Cergy : CRTF.

CAVAlli, Marisa \& Coste, Daniel. (2015). Éducation, mobilité, altérité. Les fonctions de médiation à l'école. Strasbourg : Conseil de l'Europe.

CONSEIL DE L'EUROPE. (2001). Un cadre européen commun de référence pour les langues : apprendre, enseigner, évaluer. Paris : Didier.

COSTE, Daniel, Moore, Danièle \& ZARATE, Geneviève. (1997). Compétence plurilingue et pluriculturelle. Strasbourg : Conseil de l'Europe.

DOMPMARTIN-NoRmAND, Chantal. (2016). Écrivains plurilingues et étudiants de FLE. Carnets : revue électronique d'études françaises, II(7), 234-250.

Mbodj-Pouye, Aissatou \& VAn den Avenne, Cécile. (2007). « C'est bambara et français mélangés ». Analyser des écrits plurilingues à partir du cas de cahiers villageois recueillis au Mali. Langage et Société, 120, 99-127.

MOORE, Danièle. (2005). Rencontres graphiques, pratiques cross-scripturales et mondes imaginés. Synergie France, 3, 58-75.

SCOTTISH GOVERNMENT. (2012). Language Learning in Scotland. A $1+2$ Approach. Report and recommendations. Disponible en ligne sur <www.gov.scot/resource/0039/00393435.pdf> (dernière consultation le 9 janvier 2018). 


\section{NOTES}

1. Voir les poèmes lauréats : <www.scilt.org.uk/Portals/24/Library/MTOT/2015-16/Resources/ MTOT\%202015-16\%20anthology_FINAL\%20V2.pdf> (dernière consultation le 9 janvier 2018)

2. Nous reviendrons en détails sur ce qui est entendu par " poème ».

3. Le CECRL évoque la traduction, le compte rendu, le résumé et l'interprétariat comme exemples d'activités de médiation.

4. Détaillés en partie 3 .

5. Les entretiens ont été réalisés en anglais avec la présence de l'enseignante. Tous les élèves rencontrés avaient un niveau oral en anglais suffisamment développé pour s'exprimer au sujet de leurs expériences.

6. SI. - «Je me rappelle ce jour-là après que Miss $\mathrm{B}^{*}$ me l'a dit je suis rentrée à la maison et je l'ai dit à ma mère et elle m'a fait tu te rends comptes/ euh c'est la première fois que tu fais ça et j'étais là mais je n'ai jamais entendu parler de cette compétition j'avais cette idée/j'étais pas trop sûre j'étais contente de pouvoir partager ma langue et j'étais contrariée parce que je n'arrivais pas à trouver de poème et puis quelques semaines plus tard ça m'est venue en tête comme ça ce poème et j'ai commencé à écrire. »

7. BL. - «Ben j'étais là ooooh mais qu'est-ce qu'on fait j'étais là c'est quoi le but de ce truc je comprends pas et j'étais là j'ai vraiment pas envie de faire ça mais je sais pas j'aime bien parce qu'on a pu dessiner et tout (rires) mais ensuite j'ai commencé à aimer alors c'est bien/ Mais au début j'aimais pas "

M. - « Et alors qu'est-ce qui t'a fait changer d'avis?»

BL. - «Ben j'ai appris qu'il faut juste qu'on s'exprime et pas juste faire un dessin faire un poème qui en fait veut dire quelque chose»

M. - «Hmm»

BL. - « [j'ai] commencé à apprécier les poèmes c'est ça qui a changé »

8. SI. - «J'ai trouvé ça super parce qu'en fait c'était la première fois que j'écrivais dans ma propre langue et je me rappelle j'étais à la maison et ma mère était dans la chambre et je pensais utiliser des syllabes pour écrire et j'ai fait maman est-ce que c'est juste et elle m'a fait comment tu sais écrire ça et je lui ai dit j'ai juste utilisé des syllabes »

LU. - «C'est pareil pour ma langue parce que personne ne sait comment l'écrire chez moi [...] du coup je me suis dit pourquoi pas simplement utiliser des lettres anglaises pour écrire les mots »

9. « ma mère n'arrêtait pas de me gronder en me disant de le faire ».

10. SI. - « [les gens dans la classe] étaient là vous allez où je leur ai dit c'est ce projet sur les gens qui parlent plus d'une langue du coup ils étaient là ah c'est cool et j'étais là pourquoi vous n'en feriez pas partie et ils étaient là oh parce que je parle pas une autre langue mais je leur disais vous vous rendez compte qu'on apprend l'espagnol à l'école vous pourriez trouver un poème espagnol mais ils étaient là non j'ai pas envie et ils étaient là mais c'est super parce qu'on ne t'avait jamais entendu parler ta langue alors avoir la chance de t'écouter réciter ton poème ça c'est super. »

11. KE1. - «Toujours les gens me demandent de parler dans ma langue mais je n'ai pas envie parce que j'ai peur »

K. - « Tu as peur de quoi?»

KE1. - «Parce que les gens pensent c'est quoi/ juste/ tu sais/ enfin [...] tu demandes en anglais au lieu de ta langue c'est juste/ tu as peur [...]»

K. - «Et avec nous ici?»

KE1. - « J'ai confiance ici parce que c'est les gens mais en classe non » 
12. M. - «C'était comment de parler de l'Edo et de parler en Edo à l'école?»

LU. - «C'est... juste normal [...] comme parler polonais [...] je sais pas, parler polonais à l'école genre si tu es polonais c'est... c'est juste normal pour moi [...]»

13. SO. - «Ça n'a rien de différent/ genre si tu es français par exemple et que tu n'as jamais parlé anglais et puis tu viens habiter ici et tu parles anglais ben/ en France ils seraient là oh estce que c'est différent mais ça ne l'est pas [...]»

14. Une communication à ce sujet a été présentée en janvier 2017 à l'université Bordeaux Montaigne lors du colloque ACEDLE, «La médiation en didactique des langues : formes, fonctions, représentations ".

15. Traduction du texte explicatif :

«Mon poème est une comptine à propos d'un perroquet assis sur un arbre à anone. Cette comptine est très populaire auprès des enfants du Bengladesh. L'anone est un fruit d'Inde et est un des fruits préférés de ma mère. Ma mère me racontait cette comptine quand j'étais plus jeune avant d'aller dormir et quand on va voir ma grand-mère au Bengladesh, elle me la raconte aussi.

Ma partie préférée du poème est quand le perroquet appelle la mariée. Quand [j'entends] ce poème cela me fait penser au perroquet assis sur son arbre. Je me demande si le perroquet chante quelque chose à la mariée. »

16. Selon Mbodj-Pouye et Van den Avenne (2007), la translittération désigne « l'acte d'écrire dans une graphie distincte de celle qui est originellement associée à la langue du texte » (p. 102).

17. Pratiques d'écriture sans contraintes, de loisirs, ne se limitant pas aux usages normés de la langue.

18. Expression calquée sur son équivalente en anglais creative writing.

\section{RÉSUMÉS}

Mother Tongue Other Tongue est un concours de poésie ouvert à toutes les langues proposées dans les écoles primaires et secondaires en Écosse. Une enquête a été réalisée lors de l'édition 2015-2016 par le biais d'entretiens oraux semi-directifs dans quatre écoles ayant participé et par la constitution d'un corpus de 97 productions bilingues (anglais/autre langue). La dimension individuelle et personnelle de ce projet impose à l'élève un travail de réflexion sur son rapport aux langues. La dimension collective de ce projet et son objectif de diffusion auprès d'un large public influent également de manière significative sur ce travail. Ainsi, nous verrons comment cette expérience, à travers le processus d'autonomisation qu'elle implique et le travail de médiation que l'enfant doit réaliser, se présente comme une opportunité d'appropriation ou de réappropriation linguistique pour l'enfant.

Mother Tongue Other Tongue is a poetry competition open to all languages in Scottish primary and secondary schools. Over the course of its 2015-2016 edition, semi-guided interviews were conducted with children who participated in the competition from four primary schools and 97 bilingual productions (English/other language) were retained as a corpus. The individual and personal dimension of this project encourages the child to think about how he/she relates to his/ her languages. The collective dimension of the project and its aim of public promotion also impact this personal reflection. We will show how this experience-which involves a process of autonomy and a mediation activity-allows in itself an opportunity for linguistic appropriation or reappropriation. 
INDEX

Mots-clés : pratiques plurilingues, poésie, écriture créative, enfants

Keywords : poetry, children, creative writing, multilingualism

\section{AUTEUR}

MALIKA PEDLEY

Doctorante IKER UMR 5478, Université Bordeaux Montaigne 